\title{
Spatially Complete Global Spectral Surface Albedos: Value-Added Datasets Derived from Terra MODIS Land Products
}

\author{
Eric G. Moody, Michael D. King, Senior Member, IEEE, Steven Platnick, \\ Crystal B. Schaaf, Member, IEEE, and Feng Gao, Member, IEEE
}

IEEE Transactions on Geoscience and Remote Sensing

Manuscript submitted April 21, 2004.

E. G. Moody is with L-3 Communications Government Services, Inc., Vienna, VA 22180 USA (e-mail: moody@climate.gsfc.nasa.gov).

M. D. King and S. Platnick are with the Earth Sciences Directorate, NASA Goddard Space Flight Center, Greenbelt, MD 20771 USA.

C. B. Schaaf and F. Gao are with Center for Remote Sensing, Department of Geography, Boston University, Boston, MA 02215 USA. 
Popular Summary - Land surface albedo is an important parameter in describing the radiative properties of the earth's surface as it represents the amount of incoming solar radiation that is reflected from the surface. The amount and type of vegetation of the surface dramatically alters the amount of radiation that is reflected; for example, croplands that contain leafy vegetation will reflect radiation very differently than blacktop associated with urban areas. In addition, since vegetation goes through a growth, or phenological, cycle, the amount of radiation that is reflected changes over the course of a year. As a result, albedo is both temporally and spatially dependant upon global location as there is a distribution of vegetated surface types and growing conditions.

Land surface albedo is critical for a wide variety of earth system research projects including but not restricted to remote sensing of atmospheric aerosol and cloud properties from space, ground-based analysis of aerosol optical properties from surface-based sun/sky radiometers, biophysically-based land surface modeling of the exchange of energy, water, momentum, and carbon for various land use categories, and surface energy balance studies. These projects require proper representation of the surface albedo's spatial, spectral, and temporal variations, however, these representations are often lacking in datasets prior to the latest generation of land surface albedo products.

Recent production of land surface anisotropy, diffuse bihemispherical (white-sky) albedo and direct beam directional hemispherical (black-sky) albedo from observations acquired by the Moderate Resolution Imaging Spectroradiometer (MODIS) instruments aboard NASA's Terra and Aqua satellite platforms have provided researchers with unprecedented spatial, spectral, and temporal information on the land surface's radiative characteristics. Cloud cover, which curtails retrievals, and the presence of ephemeral and seasonal snow limit the 
snow-free data to approximately half the global land surfaces on an annual equal-angle basis. This precludes the operational MODIS albedo products from being used in some earth system research projects.

To provide complete representation, an ecosystem-dependant temporal interpolation technique has been developed to fill missing or seasonally snowcovered data in the official albedo product. The method imposes pixel-level and local regional ecosystem-dependent phenological behavior onto retrieved pixel temporal data in such a way as to maintain pixel-level spatial and spectral detail and integrity. The phenological curves are derived from statistics based on the International Geosphere-Biosphere Programme (IGBP) land cover classification product geolocated with the albedo data. The resulting snow-free value-added products provide the scientific community with spatially and temporally complete global white- and black-sky surface albedo maps and statistics. These products are stored on one-minute and coarser resolution equal-angle grids, and are computed for the first seven MODIS wavelengths, ranging from 0.47 through $2.1 \mu \mathrm{m}$, and for three broadband wavelengths, 0.3-0.7, 0.3-5.0 and 0.7-5.0 $\mu \mathrm{m}$. 